January 2006

\title{
Effects of dorsiflexor endurance exercises on foot drop secondary to multiple sclerosis: A pilot study
}

Julie Mount

Thomas Jefferson University

Stan Dacko

Lebanon Valley College

Follow this and additional works at: https://jdc.jefferson.edu/ptfp

Part of the Physical Therapy Commons

Let us know how access to this document benefits you

\section{Recommended Citation}

Mount, Julie and Dacko, Stan, "Effects of dorsiflexor endurance exercises on foot drop secondary to multiple sclerosis: A pilot study" (2006). Department of Physical Therapy Faculty Papers. Paper 1.

https://jdc.jefferson.edu/ptfp/1

This Article is brought to you for free and open access by the Jefferson Digital Commons. The Jefferson Digital Commons is a service of Thomas Jefferson University's Center for Teaching and Learning (CTL). The Commons is a showcase for Jefferson books and journals, peer-reviewed scholarly publications, unique historical collections from the University archives, and teaching tools. The Jefferson Digital Commons allows researchers and interested readers anywhere in the world to learn about and keep up to date with Jefferson scholarship. This article has been accepted for inclusion in Department of Physical Therapy Faculty Papers by an authorized administrator of the Jefferson Digital Commons. For more information, please contact: JeffersonDigitalCommons@jefferson.edu. 


\title{
Effects of Dorsiflexor Endurance Exercises on Foot Drop Secondary to Multiple Sclerosis: A Pilot Study
}

\author{
Julie Mount \\ Stan Dacko
}

Julie Mount, PT, PhD is Associate Professor, Department of Physical Therapy, College of Health Professions, Thomas Jefferson University, 830 Edison Bldg., 130 S. $9^{\text {th }}$ St., Philadelphia, PA 19107. julie.mount@jefferson.edu. Telephone: 215 503-6017. FAX: 215 503-3499. Address all correspondence to Dr. Mount.

Stan Dacko, PT, PhD is Associate Professor, Department of Physical Therapy,Lebanon Valley College, 101 N. College Ave., Annville, PA 17003..

This study was supported by a grant from the National Multiple Sclerosis Society.

This study was approved by the Institutional Review Board at Thomas Jefferson University.

Keywords: multiple sclerosis, exercise, foot drop 


\begin{abstract}
PURPOSE: This is a pilot study to determine if endurance exercises for dorsiflexors will improve walking for people with foot drop secondary to MS, and if improvement in muscle endurance for persons with MS can be predicted based on the amount of central fatigue (CF) in the muscle.
\end{abstract}

SUBJECTS: Five individuals with foot drop secondary to MS and five age-matched controls. METHODS: The intervention was 4 sets of 10 isometric contractions, at $60 \%$ of MVC, 3X/week, 8 weeks. Pretests-posttests included a dorsiflexor endurance test with superimposed electrical stimulation to measure $\mathrm{CF}$, and a gait assessment including measurement of dorsiflexion at initial contact.

RESULTS: 2 of 5 subjects with MS had significant improvement in muscle endurance in both legs and in dorsiflexor control during walking. Correlation between $\mathrm{CF}$ and improved endurance was not significant $(\mathrm{r}=-0.07)$. One of the subjects with significant improvement in endurance had a significant decrease in central activation failure.

CONCLUSIONS AND DISCUSSION: For individuals with foot drop secondary to MS, endurance exercises for the dorsiflexors can result in improved walking. CF may not be useful in predicting whether a muscle will improve in endurance with exercise. Improvement may result from either a peripheral training effect, a central learning effect, or both. 
Muscle fatigue has been identified as a phenomenon distinct from muscle weakness in people with multiple sclerosis [11]. Muscle fatigue, which can also be described as poor muscle endurance, can result in functional problems. One example of a functional deficit that is clearly caused by muscle fatigue is the development of foot drop after walking some distance. When a patient presents with a disability caused by poor muscle endurance, one intervention that could be offered would be a muscle endurance exercise program. There is inadequate research available to determine whether a muscle endurance exercise program will improve function for a person with multiple sclerosis.

Muscle fatigue in individuals with MS could be caused by changes in the ability of the central nervous system to activate the muscles due to the pathology, or it could be caused by peripheral changes in the muscles secondary to decreased activity level. A decrease in the force-generating capacity of a muscle with repeated contractions caused by failure of neural drive to the muscle is called central fatigue (CF). Several studies provide evidence that the muscles of people with MS have CF. Latash et al.[7] found that when electrical stimulation was superimposed over voluntary contractions of the quadriceps muscle of people with multiple sclerosis, that there was often a greater increase in torque with stimulation as the muscle fatigued. This phenomenon was not observed in healthy control subjects. The increase in torque caused by superimposition of electrical stimulation over a maximum voluntary contraction indicates that the muscle has the capacity to generate more torque, but voluntary muscle performance is limited by problems with central activation. Sheean et al [12] used a similar methodology with the adductor pollicis muscle and also found evidence of central fatigue for subjects with MS. Kent-Braun et al.[4] 
found, using superimposed electric stimulation on the dorsiflexor muscles of 6 subjects with MS, that 3 had central fatigue and the other 3 did not. If muscle performance is limited directly by the pathology of MS, it is not clear whether it will be improved by an endurance exercise program.

On the other hand, numerous studies have shown that people with MS are likely to have peripheral changes due to deconditioning. $[5,6,8,14]$ If poor muscle endurance is due to deconditioning, one would expect improved endurance as a result of participation in an endurance exercise program. Peripheral fatigue $(\mathrm{PF})$ is the term used to describe the decrease in the force-generating capacity of a muscle with repeated contractions caused by changes within the muscle that are typically associated with deconditioning.

Given the variability in the location of the sclerotic plaques and the pattern of decreased activity among individuals with MS, it would not be surprising to find varying degrees of central and peripheral fatigue in each muscle. It is possible that the amount of improvement in muscle endurance that an individual with MS can achieve is dependent on the degree to which the activation of that particular muscle is limited by central fatigue caused by MS.

Only three studies were found in the literature that addressed the effect of exercise on muscle endurance/ fatigue in people with MS. One study, which reported only averages across the 10 subjects, reported improved endurance among leg muscles but not arm muscles [2]. The other two studies reported that some subjects with MS experienced improved muscle endurance with exercise, and others did not. $[13,10]$. One of the studies noted a trend that those with more severe disability were least likely to gain from exercise [10].A recent systematic literature review 
concluded that for people with MS, exercise improves exercise tolerance functions as well as muscle power and mobility-related activities [9]. However, previous studies of the effect of endurance exercises on people with MS did not identify whether the muscles that were found to have improved had been directly affected by MS. If individuals who are more disabled are less likely to improve, as Shapiro reported, [10] then one might expect that parts of the body that are displaying muscle fatigue secondary to MS that is severe enough to interfere with daily activities will be less likely to improve with exercise, and yet poor muscle endurance that interferes with daily function is exactly the type of impairment for which a patient is likely to seek physical therapy. Undertaking an endurance exercise program takes time, effort, commitment, and resources. It would be useful to be able to predict for an individual with MS whether he or she is likely to improve with exercise.

There were several purposes of this pilot study: 1) to determine if exercise would result in improvement in endurance for muscles that have severe enough fatigue secondary to MS that they limit daily activities;2) to determine if improvement in muscle endurance through isolated exercise of the dorsiflexors would carry over to improved walking among individuals with MS who report that their walking distance was limited by foot drop; and 3) to determine if the amount of CF in the muscle, as determined by superimposed electrical stimulation during a muscle endurance test, could be used to predict whether a client's muscle endurance can be substantially improved through participation in a muscle endurance exercise program. We hypothesized that subjects who have a greater proportion of central fatigue would have less improvement in muscle endurance and function following a muscle endurance exercise program. 


\section{Methods}

Subjects

Five individuals with multiple sclerosis who reported the onset of foot drop in a least one foot after walking some distance participated. Inclusion criteria were that they must be able to walk at least 10 meters with or without assistive devices, but without an ankle-foot orthosis or human assistance, and they must be able to give informed consent. Subjects were excluded if they had an exacerbation within the three months prior to the study, if they were unable to generate a measurable force with their dorsiflexors or achieve neutral dorsiflexion passively, or if they did not have at least 10 degrees of passive excursion of the ankle. The procedure was performed on both legs of each of the five subjects. The subjects with MS included 2 females (ages 42 and 55) and 3 males (ages 37, 52, and 59). Other information about the subjects with MS is provided in Table 1.

Five individuals with no known neurologic pathology and with no ankle impairments, who matched the subjects with MS in terms of age and gender, were recruited. There were three purposes for including healthy controls: to determine if healthy individuals could fully activate the tibialis anterior muscle performing this protocol, to test the reliability of the torque measurements on the Biodex when our protocol was used,and to compare the magnitude of change in muscle endurance for subjects with MS to that achieved by individuals without MS. One of the control subjects had to quit before completing the study due to job relocation. All subjects signed an informed consent form approved by the Institutional Review Board. 


\section{Instrumentation}

The Ashworth Scale of Muscle Spasticity [1] and the Incapacity Status Scale (ISS) [3] were used to collect descriptive information about the subjects with MS (see table 1). A Biodex MultiJoint System 3 Isokinetic Assessment System * was used for the exercise program and for the muscle endurance testing. An Intelect Model 500 Stimulator $^{\dagger}$ was used to identify the motor points of the muscles for each subject. A Grass electrical stimulation unit model \#S8800 and isolation unit model \#S1U8T ${ }^{\ddagger}$ were used to provide electrical stimulation to the anterior tibialis during the muscle endurance testing.

For the walking protocol, an electrogoniometer was taped and strapped to the more involved leg, aligned for measurement of ankle dorsiflexion, and footswitches were taped to the plantar surfaces of the heel, and the medial and lateral forefoot. Information from the electrogoniometer and 3 footswitches were synchronized through the Tattle Tale Palo Alto Data Acquisition System (TTPADAS) ${ }^{\S}$ which is a lightweight untethered system for collecting and storing data with a sampling rate of $33 \mathrm{hz}$. The angle of the ankle was calibrated using a standard goniometer.

*Biodex Model \#: Pro 3, Biodex Medical Systems, Inc. Brookhaven R \& D Plaza, 20 Ramsay Rd. Box 702, Shirley, NY 11967-0702.

${ }^{\dagger}$ Intelect Model 500, Chattanooga Corporation, Chattanooga, Tennessee 37405.

${ }^{\ddagger}$ Grass Stimulator Model \# S8800 and Isolation Unit Model \# S1U8T. Grass Instrument Division, Astromed, Inc. 600 East Greenwich Ave., West Warwick, RI 02893.

${ }^{\S}$ Tattle Tale Palo Alto Data Acquisition System (TTPADAS), developed by Eric Sableman, PhD, VA Medical Center, Rehabilitation Research \& Development, Palo Alto, CA 94304. 


\section{Design and Procedure}

A pretest-posttest design was used. On the initial visit, subjects were asked questions about the history of their disease, whether they had used an AFO, and how far they could walk before they had to stop because of their foot drop. The ISS was administered. Passive range of motion, muscle strength and spasticity were measured.

Pretests and posttests for the healthy control subjects consisted of 5 sessions of the muscle endurance protocol described below. Performance was tested 5 different times in order to capture the variability that occurred for each subject to enable a single subject design to be used for evaluating changes. Pretests and posttests for the subjects with MS consisted of 5 sessions of the muscle endurance protocol and 5 sessions of the walking protocol described below. An attempt was made to alternate walking and muscle endurance protocols so that these tests were performed over the same time span; however, occasional deviations from the alternation pattern occurred due to problems with availability of the testing area. At least 48 hours of recovery time was allowed between any two pretests or posttests.

\section{Muscle Endurance Protocol}

Muscle endurance testing was performed isometrically with the ankle at $5^{\circ}$ of plantarflexion. An ICC was used to determine the reliability of this set up for measuring dorsiflexor torque output, based on the 5 pretest values for each leg of the healthy control subjects. The reliability was found to be .92 . Each subject was given two trials of 5 second maximum voluntary contractions (MVC) with a three minute rest in between. Electrodes $2.8 \mathrm{~cm}$ in diameter were 
applied to the anterior tibialis. The Grass stimulator was set to emit a $10 \mathrm{~ms}$ train of $0.6 \mathrm{~ms}$ pulses at $100 \mathrm{hz}$. Electical stimulation was applied to the anterior tibialis with a 60 second rest between each stimulation. Intensity was gradually increased until the torque output stopped rising with an increase in intensity. The intensity was set one unit above the point at which the torque plateaued. After another 3 minute rest, the subject was asked to perform a third MVC, and during this MVC a supramaximal stimulation was applied.

After another 3 minute rest, the endurance test was initiated. A target line was placed on the screen representing $60 \%$ of the highest MVC the subject produced that day. The subject was asked to perform repeated isometric contractions of the dorsiflexors at $60 \% \mathrm{MVC}$ with a 7 second on, 3 second off cycle. When 2 consecutive repetitions occurred in which the subject was not able to achieve the $60 \%$ target for at least 0.5 seconds, the subject was asked to generate an MVC. During each MVC, electrical stimulation was superimposed at the previously established supramaximal intensity. Torque output was recorded continuously on the Biodex. For the posttests, $60 \%$ of the mean pretest MVC for each leg was used as the target.

\section{Walking Endurance Protocol}

The distance each subject could walk was measured on 5 separate days pre-intervention and 5 separate days post-intervention. Subjects were instructed to walk at their usual pace, and to inform the investigator walking beside them when they started developing symptoms that would ordinarily cause them to stop walking, such as fatigue, or feeling unstable due to foot drop. An electrogoniometer and footswitches were used to record the amount of dorsiflexion at the point 
that the foot made initial contact with the floor for each step. Time spent walking was recorded using a stopwatch.

\section{Exercise Protocol}

Each subject underwent an 8 week program, exercising the anterior tibialis of each leg 3 times a week using the following protocol. The highest MVC of 3 attempts for the day was recorded at the beginning of each session for each leg. A target line was placed on the feedback monitor of the Biodex indicating a torque output $60 \%$ of the maximum for that day. Subjects then performed 10 repetitions of isometric dorsiflexion, with the ankle in $5^{\circ}$ of plantarflexion, with visual feedback to maintain a torque output at $60 \% \mathrm{MVC}$ for 7 seconds followed by a 3 second rest. A total of 4 sets of 10 repetitions, with 2 minute rest breaks between sets, were performed during each session.

\section{Data Analysis}

Muscle endurance was defined as the number of repetitions the subject could perform before s/he was unable to achieve at least $60 \%$ of the maximum voluntary contraction (MVC) achieved on the pretest for at least 0.5 seconds for two consecutive repetitions. Improvement in muscle endurance was the difference between the mean number of repetitions performed on pretest and the mean number of repetitions performed on the posttests after the 8 week exercise program. Wilcoxon rank sum tests were done on the number of repetitions successfully performed pretest 
vs post-test for each subject to determine if there was significant improvement in muscle endurance for that subject.

Central activation failure $(C A F)$ was defined as (force increment/stimulated MVC force) X 100, where the stimulated MVC force was the maximum amount of force produced when a supramaximal electrical stimulation (SES) was superimposed over a maximum voluntary contraction (MVC), and the force increment was the difference between the stimulated MVC force and the maximum force that the subject could produce voluntarily in the absence of electrical stimulation. During each test session CAF was determined during the initial MVC and again when the subject had fatigued. Central Fatigue $(C F)$ was the increase in central activation failure that occurred when the muscle exercised to the point of fatigue. The point of fatigue was determined by the inability to achieve $60 \%$ of the baseline MVC for at least 0.5 seconds for two successive repetitions. The relationship between $\mathrm{CF}$ and improvement in muscle endurance was tested with a Spearman correlation coefficient.

Changes in distance that a subject could walk before needing a rest were assessed for each subject using Wilcoxon rank sum tests. The synchronized records of the footswitches and electrogoniometer were used to determine the angle of the ankle at the point of initial contact for each step. Differences between pretest and posttest angles of the ankle at initial contact were evaluated for each subject separately using t-tests. The significance level for all tests was set at $\alpha=.05$. 


\section{Results}

Table 2 shows the pretest and posttest MVC's expressed as a percentage of bodyweight and the change in the MVC from pretest to posttest. It also shows the pretest and posttest endurance in \# of repetitions and the change in the \# of repetitions from pretest to posttest. An asterisk indicates that the change was significant based on a two-tailed Wilcoxon rank sum test.

The strength of the dorsiflexors, as measured by the MVC, was significantly greater for the age and gender matched controls in every case but one. The exception was the female in her mid 50 's. While the exercise protocol was designed to increase endurance rather than strength, there was a significant improvement in strength in two legs of persons with MS, and in one leg of a healthy control subject.

Endurance improved in 5 out of 8 legs of control subjects. The male control subject in his 50's experienced muscle cramping during some of the post-test sessions, causing him to decrease his torque output prematurely on those days. The female control subject in her 50's had a substantial increase in her mean number of repetitions in the right leg, but the difference was not significant because of the variability in her performance. Of the 5 subjects with MS, 2 had dramatic improvement in the endurance of both their leg with foot drop and their leg without foot drop, and the other 3 had no significant change in the endurance of either leg.

Table 3 shows the initial CAF, the amount of CAF when the subject's muscle had been fatigued to the point of not being able to voluntarily generate $60 \%$ of the muscle's MVC, and the CF. 
These values are reported at pretest and posttest. The change from pretest to posttest is reported, and an asterisk is used to indicate if the change is significant. None of the control subjects had $\mathrm{CAF}$, and therefore they are not included in this table. All of the subjects with MS had some amount of central activation failure in their leg with foot drop and, in most cases, their leg without foot drop. One subject with MS demonstrated a significant decrease in CAF from pretest to posttest, and another subject demonstrated a significant increase in CAF from pretest to posttest in the involved leg. In a few cases CF was negative, indicating that the subject had more difficulty fully activating the leg at the beginning of a bout of exercise than when fatigued.

For the dorsiflexors of subjects with MS, there was not a significant correlation between pretest $\mathrm{CF}$ and improvement in endurance $(\mathrm{r}=-0.07)$. There was also not a significant correlation between pretest initial CAF and improvement in endurance $(r=0.12)$.

Data from the walking protocol are presented in table 4. Subjects MS2 and MS3 had significant improvement in their walking endurance, and MS2 also had significant improvement in his walking velocity as measured during the pretests and posttests. MS2 reported that he had increased the number of holes he could walk on the golf course. Although MS1 did not significantly increase his walking distance as measured on the indoor track, he reported that his community walking distance increased from 3 blocks to 6 blocks. Analysis of the data from the electrogoniometers and footswitches indicated that MS1 and MS2 had significant increases in the amount of dorsiflexion in the FD leg at initial contact. Other subjects had differences in dorsiflexion at initial contact that were less than $2^{\circ}$, which we judged to be clinically insignificant. 


\section{Discussion}

The results of this study suggest that for some individuals with MS, muscle endurance can improve with exercise despite the fact that MS is reducing the patient's ability to centrally activate the muscle. On the other hand, 3 of the 5 subjects in this study did not experience significant improvement with exercise. One purpose of this study was to determine if good candidates for an exercise program could be predicted based on a test that could be performed prior to initiating the exercise program. We hypothesized that muscles with large amounts of central fatigue, indicating that the poor endurance was due to central factors, would not improve with a standard muscle endurance exercise program. This hypothesis was not supported by the data. Some subjects had a high proportion of CAF on their initial unfatigued MVC's, which would indicate that activation of the muscle was limited by the pathology of MS, even though they did not have very much CF. There was no correlation between the initial CAF and the amount of improvement, suggesting that the extent to which MS was directly limiting function of the muscle did not correlate with the ability of the muscle to respond to the exercise program provided in this study.

There was a significant decrease in CAF from pretest to posttest for one subject who achieved substantial improvement in endurance of his dorsiflexors on the leg that experienced foot drop. A decrease in CAF means that the central nervous system is able to activate the muscle more fully. This suggests that either there was a remission of the disease, or the central nervous system relearned how to activate the muscle using alternate pathways. The subject had exacerbating and remitting MS, but had not experienced an exacerbation for over a year. It is 
therefore unlikely that the improvement in CNS function was a result of remission. While it is not surprising for improved function for an individual with a central nervous system disorder to occur through changes in the central nervous system, the exercise program in this study was designed to impact endurance at a peripheral level. Subjects were performing 4 sets of 10 repetitions of an isolated isometric contraction, in a nonfunctional context. However, in order to have the subjects exercise at $60 \%$ of their maximum voluntary contraction, subjects were given a target line on a monitor so that they would know how hard to pull to achieve the $60 \%$. This feedback on their performance may have enabled them to learn alternate pathways for accessing the muscle. It is possible that the availability of visual feedback of torque output was critical to the ability of subjects to learn alternate, more effective methods of activating their muscles. Future research should investigate the effectiveness of endurance training with feedback compared to endurance training without feedback for people with MS and use superimposed electrical stimulation to determine the extent to which improvement is a result of central versus peripheral changes.

Three of the four legs of subjects with MS that experienced significant improvement in endurance did not have significant changes in CAF, and the leg of MS2 that did not have foot drop actually had a significant increase in CF. These findings suggest that there are different mechanisms by which individuals with MS may increase their endurance. Some may increase their muscular endurance through a training effect that primarily reduces peripheral fatigue. Others may learn to access their muscles through alternate routes in the CNS, thereby increasing their ability to generate torque with that muscle. Some may improve by improving both central and peripheral factors. 
Our results from these 5 subjects with MS suggest that neither CF nor CAF is an adequate predictor of whether or not an exercise program will be effective. Other variables need to be considered. The fact that two subjects had significant improvement in both their leg with foot drop and their leg without foot drop, while the 3 other subjects did not achieve significant improvement in either leg, suggests that a better predictor might be a feature of the person, not of the influence of MS on a particular muscle. A review of the demographic information collected for these subjects (see Table 1) suggests a trend towards more success for subjects who were more recently diagnosed. Other variables such as gender, age, course of the disease, ISS score, muscle grade, and use of AFO do not appear to differentiate the more successful from the less successful subjects.

The two subjects who had the greatest improvements in muscle endurance also had improvements in their ability to walk. One of the subjects improved his reported community walking distance as well as walking significantly farther in the lab. The other did not improve the distance walked on the smooth surface in the lab, but doubled the distance he walked on the uneven sidewalks of center city. Both of the subjects who had significant improvements in muscle endurance showed significant improvement in the angle of their ankle during walking. The fact that the subjects who had significant improvement in dorsiflexor muscle endurance had improvements in walking performance suggests that interventions that work on isolated dorsiflexor endurance and control can carry over to functional walking. 


\section{Conclusions}

The results of this pilot study suggest that improvement in muscle endurance for people with MS can result from either peripheral or central changes, and that the degree of central fatigue in a muscle may not be a good predictor by itself of whether the individual will improve with exercise. The results also provide evidence that exercising an isolated muscle on an exercise machine can result in functional improvements in walking for people with foot drop secondary to MS. Further research is needed to determine the best predictors of who will benefit from exercise and who will not, who will improve as a result of central changes and who will improve as a result of peripheral changes, and what type of intervention is optimal for achieving central changes vs. peripheral changes. 


\section{Acknowledgements}

We would like to thank the following individuals for their assistance with this research: Dr.

Marcus Besser, Ann deGruy, Kimberly Fisher, Jill Fisher, Mary Frady, Angela Gordon,

Kimberly Lesser, Dina Robbins, Yvonne Spinelli, Amy Swing, Diana Taylor 


\section{References}

[1] B. Ashworth, Preliminary trial of cansoprodal in multiple sclerosis, Practitioner 192 (1964), 540-542.

[2] G. M. Gehlsen, S.A. Grigsby, D.M. Winant, Effects of an aquatic fitness program on the muscular strength and endurance of patients with multiple sclerosis, Physical Therapy 64(5) (1984), 653-657.

[3] International Federation of Multiple Sclerosis Societies. Minimal Record of Disability for Multiple Sclerosis. National Multiple Sclerosis Society; 1985.

[4] J.A. Kent-Braun, K.R. Sharma, R.G. Miller, M.W. Weiner, R.G. Miller. Effects of exercise on muscle activation and metabolism in multiple sclerosis, Muscle \& Nerve 17 (1994), 11621169.

[5] J.A. Kent-Braun, K.R. Sharma, R.G. Miller, M.W. Weiner. Postexercise phosphocreatine resynthesis is slowed in multiple sclerosis. Muscle \& Nerve, 17:835-841, 1994.

[6] J.A. Kent-Braun, A.V. Ng, M. Castro, M.W. Weiner, D. Gelinas, G.A. Dudley, R.G. Miller, Strength, skeletal muscle composition, and enzyme activity in multiple sclerosis, J. Appl. Physiol. 83(6) (1997), 1998-2004.

[7] M. Latash, E. Kalugina, J. Nicholas, C. Orpett, D. Stefoski, F. Davis. Myogenic and central neurogenic factors in fatigue in multiple sclerosis, Multiple Sclerosis 1 (1996), 236-241.

[8] A.V. Ng, J.A. Kent-Braun, Quantitation of lower physical activity in persons with multiple sclerosis, Medicine and Science in Sports and Exercise 29 (1997), 517-523.

[9] M.B. Reitberg, D. Brooks, B.M.J. Uitdehaag, G. Kwakkel. Exercise therapy for multiple sclerosis. Cochrane Database of Systematic Reviews. 2, 2005.

[10] R.T. Schapiro, J.H. Petajan, D. Kosich, B. Molk, J. Feeney. Role of cardiovascular fitness in multiple sclerosis: a pilot study, J. Neuro. Rehab. 2 (1988), 43-49.

[11] S.R. Schwid, C.A, Thornton, S. Pandya, K.L. Manzur, M. Sanjak, M.D. Petrie, M.P. McDermott, A.D. Goodman. Quantitative assessment of motor fatigue and strength in MS. Neurology 1999; 53(4): 743-750.

[12] G.L. Sheean, N.M. Murray, J.C. Rothwell, D.H. Miller, A.J. Thompson, An electrophysiological study of the mechanism of fatigue in multiple sclerosis, Brain 120 (1997), 299-315. 
[13] B. Svensson, B. Gerdlee, J. Elert, Endurance training in patients with multiple sclerosis: five case studies. Physical Therapy 74(11) (1994), 1017-1026.

[14] C.A. Vaz Fragosa, D. Wirz, J. Mashman, Establishing a physiological basis to multiple sclerosis-related fatigue: a case report, Arch Phys Med Rehabil. 76 (1995), 583-586. 
Table 1. Characteristics of the five subjects with multiple sclerosis.

\begin{tabular}{|c|c|c|c|c|c|}
\hline & MS1 & MS2 & MS3 & MS4 & MS5 \\
\hline Gender & $M$ & $\mathrm{M}$ & $M$ & $\mathrm{~F}$ & $\mathrm{~F}$ \\
\hline Age & 37 & 52 & 59 & 55 & 42 \\
\hline ISS score & 4 & 4 & 7 & 3 & 16 \\
\hline Yrs. Since Dx. & 7 & 9 & 13 & 13 & 12 \\
\hline Course of MS & Exac \& Remit. & Progressive & Exac \& Remit. & Progressive & Secondary Prog. \\
\hline $\begin{array}{l}\text { Ashworth score } \\
\text { for Plantarflexors }\end{array}$ & $\begin{array}{ll}\mathrm{R} & 0 \\
\mathrm{~L} & 0\end{array}$ & $\begin{array}{ll}\mathrm{R} & 0 \\
\mathrm{~L} & 0\end{array}$ & $\begin{array}{ll}\mathrm{R} & 1 \\
\mathrm{~L} & 0\end{array}$ & $\begin{array}{ll}\mathrm{R} & 0 \\
\mathrm{~L} & 0\end{array}$ & $\begin{array}{ll}\mathrm{R} & 1 \\
\mathrm{~L} & 1\end{array}$ \\
\hline $\begin{array}{ll}\text { Dorsiflexor } & \mathrm{R} \\
\text { strength } & \mathrm{L} \\
\text { strength } & \end{array}$ & $\begin{array}{l}4 / 5 \\
5 / 5\end{array}$ & $\begin{array}{l}3 / 5 \\
5 / 5\end{array}$ & $\begin{array}{l}4 / 5 \\
5 / 5\end{array}$ & $\begin{array}{l}5 / 5 \\
4 / 5\end{array}$ & $\begin{array}{l}3+/ 5 \\
3+/ 5\end{array}$ \\
\hline Use of AFO & no & yes & yes & no & No \\
\hline Medications & $\begin{array}{c}\text { Avonex } \\
\text { Baclofen }\end{array}$ & Betaseron & None & $\begin{array}{c}\text { Baclofen, } \\
\text { Dilantin, } \\
\text { Recently } \\
\text { stopped taking } \\
\text { Betaseron }\end{array}$ & Copaxone \\
\hline
\end{tabular}


Table 2. Strength and endurance before and after muscle endurance test for subjects with and without multiple sclerosis.

\begin{tabular}{|c|c|c|c|c|c|c|}
\hline SUBJECT & $\begin{array}{l}\text { Pretest } \\
\text { MVC } \\
\text { (\% body } \\
\text { weight) }\end{array}$ & $\begin{array}{l}\text { Posttest } \\
\text { MVC } \\
\text { (\% body } \\
\text { weight) }\end{array}$ & $\begin{array}{l}\text { Change } \\
\text { in MVC } \\
\text { (\% body } \\
\text { weight) }\end{array}$ & $\begin{array}{l}\text { Pretest } \\
\text { \# Reps }\end{array}$ & $\begin{array}{l}\text { Posttest } \\
\text { \# Reps }\end{array}$ & $\begin{array}{l}\text { Change in } \\
\text { \# Reps }\end{array}$ \\
\hline MS 1 R FD & $6.7 \pm 0.6$ & $10.1 \pm 0.7$ & $+3.4^{*}$ & $.8 \pm 0.5$ & $48.0 \pm 22.7$ & $+47.2^{*}$ \\
\hline L FD & $12.0 \pm 0.4$ & $11.9 \pm 0.4$ & -0.1 & $8 . \pm 8.4$ & $41.8 \pm 17.6$ & $+33.8 *$ \\
\hline MS 2 R FD & $2.5 \pm 1.2$ & $4.0 \pm 0.6$ & +1.5 & $0.6 \pm 0.5$ & $21.8 \pm 26.5$ & $+21.2 *$ \\
\hline L FD & $9.4 \pm 06$ & $10.9 \pm 0.5$ & $+1.5^{*}$ & $17 \pm 11.1$ & $89.8 \pm 32.7$ & $+72.8^{*}$ \\
\hline MS 3 R FD & $10.5 \pm 0.8$ & $9.6 \pm 1.0$ & -0.9 & $1.0 \pm 0.0$ & $0.4 \pm 0.5$ & -0.6 \\
\hline L NFD & $12.7 \pm 0.9$ & $13.6 \pm 0.4$ & +0.9 & $5.6 \pm 5.7$ & $8.8 \pm 5.7$ & +3.2 \\
\hline MS 4 L FD & $9.1 \pm 1.9$ & $10.4 \pm 0.9$ & +1.3 & $2.0 \pm 1.6$ & $4.6 \pm 3.6$ & +4.4 \\
\hline R NFD & $11.1 \pm 0.9$ & $11.8 \pm 1.0$ & +0.7 & $7.8 \pm 4.7$ & $13.0 \pm 9.6$ & +5.2 \\
\hline MS 5 R FD & $4.0 \pm 0.7$ & $4.3 \pm 0.8$ & +0.3 & $0.8 \pm 0.4$ & $3.6 \pm 4.8$ & +2.8 \\
\hline L FD & $7.1 \pm 1.4$ & $5.5 \pm 0.6$ & -1.6 & $1.6 \pm 2.2$ & $1.8 \pm 1.1$ & +0.2 \\
\hline Control 1 R & $19.0 \pm 0.4$ & $21.5 \pm 0.5$ & $+2.5^{*}$ & $14.3 \pm 7.6$ & $29.5 \pm 2.4$ & $+15.2 *$ \\
\hline $\mathbf{L}$ & $22.1 \pm 0.2$ & $22.9 \pm 0.9$ & +0.8 & $10.7 \pm 5.5$ & $17.8 \pm 2.4$ & $+7.1 *$ \\
\hline Control $2 \mathbf{R}$ & $16.2 \pm 0.7$ & $15.9 \pm 0.8$ & -0.3 & $34.8 \pm 8.0$ & $30.8 \pm 11.1$ & -4.0 \\
\hline Control 4 R & $\begin{array}{l}15.6 \pm 0.7 \\
10.0 \pm 0.9\end{array}$ & $\begin{array}{l}16.3 \pm 1.1 \\
10.7 \pm 0.4\end{array}$ & $\begin{array}{l}+0.7 \\
+0.7\end{array}$ & $\begin{array}{l}37.4 \pm 18.2 \\
13 \pm 12.6\end{array}$ & $\begin{array}{l}44.2 \pm 14.5 \\
22.5 \pm 0.6\end{array}$ & $\begin{array}{l}+6.8 \\
+9.4\end{array}$ \\
\hline $\begin{array}{c}\mathrm{L} \\
\text { Control } 5 \text { R }\end{array}$ & $\begin{array}{l}11.7 \pm 1.8 \\
15.2 \pm 0.6\end{array}$ & $\begin{array}{l}11.9 \pm 0.6 \\
16.9 \pm 1.0\end{array}$ & $\begin{array}{l}+0.2 \\
+1.7\end{array}$ & $\begin{array}{l}9.6 \pm 4.8 \\
14.4 \pm 3.2\end{array}$ & $\begin{array}{l}21 \pm 3.5 \\
23.6 \pm 4.2\end{array}$ & $\begin{array}{l}+11.4^{*} \\
+9.2^{*}\end{array}$ \\
\hline L & $19.7 \pm 1.7$ & $20.5 \pm 0.6$ & +0.8 & $15.0 \pm 6.0$ & $24.0+3.4$ & $+9.0^{*}$ \\
\hline
\end{tabular}

$\mathrm{FD}=$ leg with observable toe drag; $\mathrm{NFD}=$ leg with no observable toe drag; * indicates significance at 0.05 level. 
Table 3. Results from muscle endurance test.

\begin{tabular}{|c|c|c|c|c|c|c|c|c|c|}
\hline SUBJECT & $\begin{array}{l}\text { Pretest } \\
\text { Initial CAF }\end{array}$ & $\begin{array}{l}\text { Posttest } \\
\text { Initial CAF }\end{array}$ & $\begin{array}{l}\text { Pretest- } \\
\text { Posttest } \\
\text { Change in } \\
\text { Initial CAF }\end{array}$ & $\begin{array}{l}\text { Pretest } \\
\text { Fatigued } \\
\text { CAF }\end{array}$ & $\begin{array}{l}\text { Posttest } \\
\text { Fatigued } \\
\text { CAF }\end{array}$ & $\begin{array}{l}\text { Pretest- } \\
\text { Posttest } \\
\text { Change in } \\
\text { Fatigued } \\
\text { CAF }\end{array}$ & $\begin{array}{l}\text { Pretest } \\
\text { Central } \\
\text { Fatigue }\end{array}$ & $\begin{array}{l}\text { Posttest } \\
\text { Central } \\
\text { Fatigue }\end{array}$ & $\begin{array}{l}\text { Pretest- } \\
\text { Posttest } \\
\text { Change in } \\
\text { Central } \\
\text { Fatigue }\end{array}$ \\
\hline MS 1 R FD & $29.3 \pm 5.4$ & $6.3 \pm 5.0$ & $-23.0^{*}$ & $50.1 \pm 6.9$ & $27.3 \pm 9.4$ & $-22.8^{*}$ & $23.0 \pm 10.5$ & $21.0 \pm 8.8$ & -2.0 \\
\hline L NFD & $0.0 \pm 0.0$ & $0.5 \pm 1.3$ & +0.5 & $4.7 \pm 10.5$ & $8.7 \pm 4.3$ & +4.0 & $4.7 \pm 10.5$ & $8.0 \pm 4.3$ & +3.3 \\
\hline MS 2 R FD & $73.0 \pm 26.6$ & $53.4 \pm 16.8$ & -19.6 & $65.6 \pm 20.9$ & $62.6 \pm 15.7$ & -3.0 & $-7.4 \pm 11.3$ & $9.3 \pm 11.5$ & +16.7 \\
\hline L NFD & $4.6 \pm 2.2$ & $5.5 \pm 3.9$ & +0.9 & $7.3 \pm 3.0$ & $19.9 \pm 4.9$ & $+12.6^{*}$ & $3.1 \pm 5.4$ & $15.2 \pm 4.3$ & $+12.1 *$ \\
\hline MS 3 R FD & $11.7 \pm 1.9$ & $32.1 \pm 12.2$ & $+20.4^{*}$ & $39.5 \pm 18.8$ & $12.1 \pm 13.5$ & $-27.4^{*}$ & $27.8 \pm 17.6$ & $-1.6 \pm$ & +29.4 \\
\hline L NFD & $2.0 \pm 1.2$ & $3.1 \pm 3.0$ & +1.1 & $9.2 \pm 2.2$ & $9.2 \pm 1.5$ & 0 & $7.2 \pm 3.1$ & $6.4 \pm 3.2$ & -0.8 \\
\hline MS 4 L FD & $9.1 \pm 11.3$ & $4.6 \pm 3.6$ & -4.5 & $3.4 \pm 3.4$ & $2.9 \pm 3.0$ & 0.5 & $0.0 \pm 0.0$ & $-0.5 \pm 2.1$ & -0.5 \\
\hline R NFD & $0.8 \pm 2.5$ & $1.1 \pm 1.0$ & +0.3 & $7.9 \pm$ & $8.2 \pm$ & +0.3 & $6.3 \pm$ & $8.2 \pm$ & +1.9 \\
\hline MS 5 R FD & $10.6 \pm 2.7$ & $10.8 \pm 3.6$ & +0.2 & $18.3 \pm 10.9$ & $17.1 \pm 7.8$ & -1.2 & $7.8 \pm 9.4$ & $6.3 \pm 7.4$ & -1.5 \\
\hline L FD & $4.1 \pm 5.8$ & $11.6 \pm 12.9$ & +7.5 & $13.7 \pm 6.3$ & $14.0 \pm 12.0$ & +0.3 & $9.6 \pm 8.4$ & $2.5 \pm 2.6$ & -7.1 \\
\hline
\end{tabular}

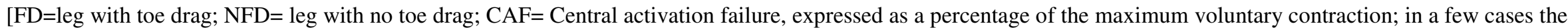

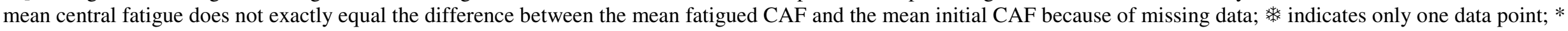
indicates significance at 0.05 level] 
Table 4. Walking data.

Pretest $\rightarrow$ Posttest difference ankle dorsiflexion at

Pretest Posttest

Subject Distance (m)

MS 1

MS 2

MS 3

MS 4

MS 5 $1109 \pm 124$

$\operatorname{Velocity}(\mathbf{m} / \mathbf{s})$ $1146 \pm 238$ $793 \pm 182 *$ $549 \pm 55 *$ $450 \pm 154$
$377+109$ $0.59 \pm 0.06$ $1069 \pm 163$

$0.83 \pm 0.05$ $0.93 \pm 0.16$ $987 \pm 191$ $166 \pm 59$ $0.60+0.12$ $231+0$ Distance (m) Velocity $(\mathbf{m} / \mathbf{s})$ $0.94+0.07$ $0.87 \pm 0.16 *$ $0.92 \pm 0.04$ $0.89 \pm 0.09$ initial contact (degrees)

$+4.2^{\circ} *$
$+5.8^{\circ} *$ $+1.3^{\circ} *$ $+1.8^{\mathrm{o}} *$ $-1.6^{\circ} *$

*Indicates a significant difference between pretest and posttest values. 